\title{
Measurement of the Dø WAMUS Magnet Inductance
}

\author{
A. Kristalinski, R. Hance and W. Jaskierny \\ Fermi National Accelerator Laboratory \\ P.O. Box 500, Batavia, Illinois 60510
}

December 1994 


\section{Disclaimer}

This report was prepared as an account of work sponsored by an agency of the United States Government. Neither the United States Government nor any agency thereof, nor any of their employees, makes any warranty, express or implied, or assumes any legal liability or responsibility for the accuracy, completeness, or usefulness of any information, apparatus, product, or process disclosed, or represents that its use would not infringe privately owned rights. Reference herein to any specific commercial product, process, or service by trade name, trademark, manufacturer, or otherwise, does not necessarily constitute or imply its endorsement, recommendation, or favoring by the United States Government or any agency thereof. The views and opinions of authors expressed herein do not necessarily state or reflect those of the United States Government or any agency thereof. 


\title{
Measurement of the DO WAMUS magnet inductance.
}

\author{
A. Kristalinski, R. Hance, W. Jaskierny \\ December 13,1994
}

\section{Introduction (Background Info on Magnet Measurements)}

Historically, the term inductance, as it relates to magnets, has been relatively obscure at Fermilab. Physicists and engineers often had different views on the subject. Confusion resulted from the typical engineering approach to the matter, whereby distinction may not have been made between analytical and beam line magnets; and distribution transformers. The latter always have a laminated core to reduce eddy currents which makes their inductance in a transitional state very close to that in a steady state. This is true only if the core material is not in saturation, which is once again the case for transformers; but not for magnets, especially the analytical ones.

Based on the traditional "transformer" thinking, an incorrect method to measure magnet inductance was initially employed. The characteristics of a tank circuit including the magnet under test were observed. Then, based on the resonant frequency and quality factor, the inductance was calculated. This method represents a very valuable tool for magnet testing where you can compare newly built magnets to a reference magnet and see if there is any difference. Although electrically correct, this method unfortunately does not reveal any valuable information which could be used to anticipate the magnet behavior under the normal working conditions. The inductance value obtained this way differs considerably from a steady state inductance due to the a very low excitation current and unsettled eddy currents.

Another method of measuring inductance, based on a freewheeling discharge of the magnet, is also widely used in the Lab. To measure the inductance, a magnet is powered from a small power supply for up to $100 \mathrm{~A}$ to $200 \mathrm{~A}$, then the power is turned off and the current decay in the magnet is recorded. Based on the dc resistance measurements and the magnet current decay data, one can determine the magnet inductance. In order to do so, the inductance is assumed to be constant and current decay is assumed to be exponential. The magnet inductance calculated this way could be called "effective" inductance. To find out how well the effective inductance represents the real process taking place in the magnet was one of the purposes of the experiment.

Inductance is essentially just a coefficient reflecting the correlation between the magnet's magnetic field and the current in the winding which produces that field. During the transient mode, the changing magnetic field creates eddy currents, which in turn generate their own magnetic field. This field reduces the expected magnetic field associated with the given current. In electrical terms it translates into a lower inductance value compared to the steady state value remaining after the eddy currents decay. The transitional inductance is lower than the real inductance value and as time passes, will rise. 
Physicists took the better approach of considering only the steady state inductance. The transition process is of little importance to a physicist who is interested in a final result - the magnetic field. The other important parameter of the magnet - the amount of the energy stored in the magnetic field - is also solely dependent upon the steady state inductance. This excludes the eddy currents from consideration. The only remaining unknown factor is the degree of saturation of the core material. This is very difficult to predict because very often the exact parameters of the core are unknown even to the magnet manufacturer. To avoid this uncertainty the core is usually assumed to be in $100 \%$ saturation. This yields reasonable results and provides data for the design of the magnets and power systems.

During the transition we can't however neglect the saturation influence. As the current rises and the core material saturates, the inductance of the magnet decreases. This leads to a very complicated transient process. As the eddy currents decay they allow the magnet inductance to rise; while at the same time the saturation of the magnetic core material tends to decrease the inductance. Thus the magnet inductance transitional behavior is very complicated and we can't adequately describe it with a simple formula. To make matters worse a very familiar formula, describing the voltage drop $V$ across the L-R circuit:

$$
V=L \cdot \frac{d i}{d t}+i \cdot R,
$$

will be transformed into the much more complicated function:

$$
V=\frac{d L}{d t} \cdot i+L \cdot \frac{d i}{d t}+i R .
$$

Consequently, we can't determine the inductance value simply by measuring the current and the voltage drop.

The design of DZERO's 2 Tesla superconducting solenoid might require the knowledge of the real inductance value of the WAMUS magnet. A few different methods were used to measure the inductance of the magnet. The WAMUS consists of three parts: the central toroid and the two end toroids. The central toroid has three air gaps. A Hall probe is installed in one of them, thus allowing the magnetic field to be actually measured. Due to the relatively small size of the air gap compared to the magnetic path in the steel, one can consider the magnetic field induction in the gap to be equal to the one in the steel. The end toroids have solid iron cores and the actual magnetic field can't be measured; but based on the muon tracking results we can assume it to be equal to 1.9 Tesla 1 . Under this assumption we will have the same error in calculating the inductance as physicists have in calculating the muon tracks; which is quite acceptable for us.

\section{Measuring the WAMUS Magnet Steady State Parameters}

First we measured the dc resistance of the system by measuring the steady state voltage drop across the magnets and buswork. The steady state current was equal to

\footnotetext{
'D0 Muon System with Proportional Drift Tube Chambers. C. Brown and others, Nuclear Instruments and Methods, A279, p.331,1989.
} 
the current value used in the muon tracking which is 2,472 Amps. The measured voltage drop was $144 \mathrm{~V}$. That yields the resistance value of .058 $\mathrm{Ohm}$.

Then we measured the voltage drop across the system while ramping the current with different constant slew rates. Our hope was to derive the inductive voltage drop by subtracting the known value of resistive voltage drop from measured voltage. Unfortunately the share of the voltage created by eddy currents, which we had hoped would be negligible, distorted the result so much that we couldn't draw reasonable conclusions with this technique.

So, we proceeded to measure the magnetic field induced in the central toroid section as a function of the current in the system. Two major results were obtained in this experiment. First of them is the fact that DO WAMUS magnet usually operates in a very deep saturation and in our opinion the current can be reduced without damaging the ability of the system to produce data, thus achieving significant savings in the operating cost of the system ${ }^{2}$. The second result was the ability to calculate center toroid inductance based on the data taken. Neglecting the leakage flux, we can calculate the toroid inductance $L_{s}$ as:

$$
L_{s}=\frac{\Psi}{I}=\frac{B \cdot N \cdot S}{I},
$$

where $\Psi$ is the magnetic flux coupled with the magnet,

$B$ is the magnetic field induction,

$N$ is the number of turns in the magnet,

$S$ is the cross section area of the magnet,

$l$ is the current in the magnet.

From ${ }^{1}$ we know that $N=200$ turns, $S=8.208 \mathrm{sq}$. $\mathrm{m}$. The measured values of $I$ and $B$ and calculated values of $L_{s}$ are shown in the Table 1 and on the Fig.1.

We know the operating current $I=2500 \mathrm{~A}$, and that the end toroids magnetic field $B=$ 1,9 Tesla. Furthermore we know the end toroid data from 1, i.e. number of turns $N=64$; cross section area $S=4.66 \mathrm{sq}$. $\mathrm{m}$. Thus we can calculate the end toroid inductance $L_{t}$ using expression (3):

$$
L_{t}=.227 \text { Henry }
$$

From these factors, we can calculate the inductance of the complete system of three toroids at $2500 \mathrm{~A} L_{\Sigma}$ as:

$$
L_{\Sigma}=L_{S}+2 L_{t}=1.27+.454=1.724 \text { [Henry] }
$$

\footnotetext{
2Energy conservation suggestion \# 151. R. Hance and others., 1994.
} 
Table 1.

\begin{tabular}{|c|c|c|}
\hline 1 & B & $L_{s}$ \\
\hline Amps & Tesla & Henry \\
\hline 100 & .500 & 8.2 \\
\hline 200 & 1.080 & 8.86 \\
\hline 300 & 1.320 & 7.22 \\
\hline 400 & 1.470 & 6.03 \\
\hline 450 & 1.520 & 5.54 \\
\hline 500 & 1.540 & 5.056 \\
\hline 550 & 1.560 & 4.656 \\
\hline 600 & 1.585 & 4.02 \\
\hline 650 & 1.605 & 4.02 \\
\hline 700 & 1.620 & 3.77 \\
\hline 750 & 1.635 & 3.61 \\
\hline 800 & 1.650 & 3.36 \\
\hline 850 & 1.665 & 3.20 \\
\hline 900 & 1.675 & 3.04 \\
\hline 950 & 1.680 & 2.95 \\
\hline 1,000 & 1.700 & 2.79 \\
\hline 1,100 & 1.722 & 2.54 \\
\hline 1,200 & 1.742 & 2.38 \\
\hline 1,300 & 1.761 & 2.22 \\
\hline 1,400 & 1.780 & 2.05 \\
\hline 1,500 & 1.795 & 1.97 \\
\hline 1,600 & 1.815 & 1.89 \\
\hline 1,700 & 1.830 & 1.72 \\
\hline 1,800 & 1.845 & 1.68 \\
\hline 1,900 & 1.860 & 1.60 \\
\hline 2,000 & 1.870 & 1.53 \\
\hline 2,100 & 1.885 & 1.47 \\
\hline 2,200 & 1.900 & 1.41 \\
\hline 2,300 & 1.910 & 1.36 \\
\hline 2,400 & 1.920 & 1.31 \\
\hline 2,500 & 1.930 & 1.27 \\
\hline
\end{tabular}




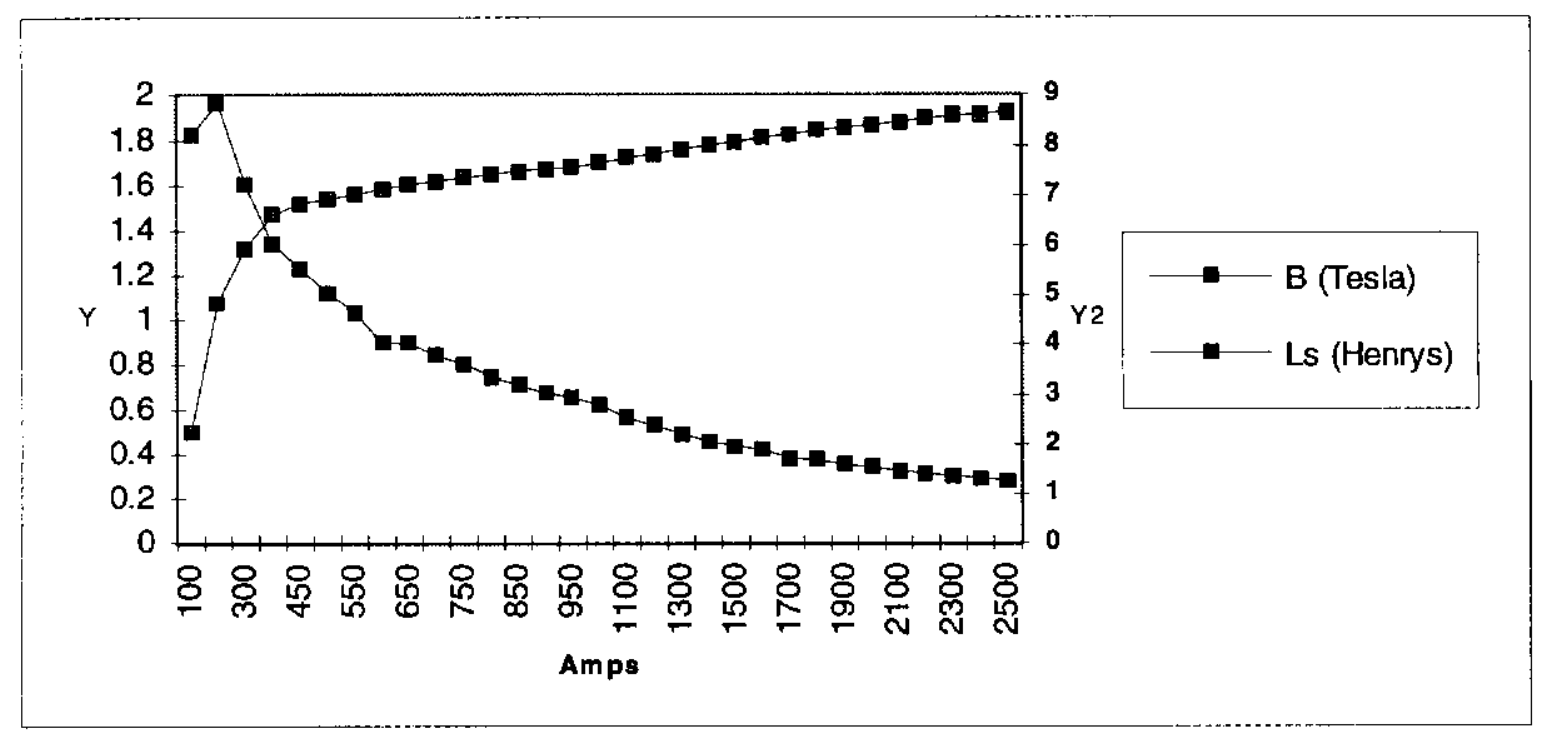

Fig. 1

\section{Measuring the WAMUS Magnet Transient Parameters}

The last experiment we did was a freewheeling discharge of the magnets as a system. The voltage across the system and the magnet current are shown in the Fig. 2 . The initial current value was $I_{O}=2500 \mathrm{~A}$. To understand how different transition processes, such as eddy currents and desaturation of the steel, affect the magnet, let's introduce the effective inductance of the system. We define the effective inductance $L_{a}$ as calculated under assumption that the current decay is exponential and the inductance is constant. We divide the actual current decay into three portions. We assume that the current decay within every portion is exponential and choose the boundaries of the portions as points between which the current value decreases by factor $e$, where $e$ is the natural logarithm base. The first portion will lie, accordingly to this definition, between $I_{0}$ and $I_{1}=I_{0} / e=920 A$. Similarly $I_{2}=I_{1} / e=338 \mathrm{~A}$, and $I_{3}=I_{2} / e=124 \mathrm{~A}$. It is known that the duration of every portion derived this way is equal to the time constant $\tau$ of the corresponding exponential function. From Fig. 1 we can find the time constants:

$$
\tau_{1}=2.8 \mathrm{sec} ; \tau_{2}=9.6 \mathrm{sec} ; \tau_{3}=28 \mathrm{sec} .
$$

Because we assumed that we are dealing with exponential current decay we can determine effective inductance $L_{a}$ as:

$$
L_{a}=\tau \cdot R
$$

From (4) we can calculate the effective inductances for all three portions of the current decay:

$$
\begin{aligned}
& L_{a 1}=.162 \text { Henry } \\
& L_{a 2}=.557 \text { Henry } \\
& L_{a 3}=1.62 \text { Henry. }
\end{aligned}
$$


The effective inductance at the first portion of the decay is ten times lower than the inductance in the steady state and remains lower than calculated steady state inductance at all times. This is caused by the energy transferred from the toroids magnetic field to the eddy current magnetic field. As a result this measurement can not be used to determine the actual inductance of the magnet.

However this result proves that the engineering method based on measurements of the current decay described in the beginning can be effectively used to predict the magnet discharge time. If we consider the actual current decay as an exponential process associated with just one effective inductance $L_{a}$, then the current decay is exponential, and current will decrease from $I_{0}$ to $I_{3}$ in the time interval $t_{1}$ equal to $3^{*} \tau_{a}$, where $\tau_{a}$ is the time constant of the exponent. $t_{1}$ is equal to the sum of $\tau_{1}, \tau_{2}$, and $\tau_{3}$, i. e. :

$$
\tau_{a}=\left(\tau_{1}+\tau_{1}+\tau_{1}\right) / 3=(2.8+9.6+28) / 3=13.5[\mathrm{sec}]
$$

From this, according to the equation (4), we can find $\mathrm{La}$;

$$
L_{a}=.058 * 13.5=.78 \text { [Henry] }
$$

This result is close to the effective inductance value, calculated on freewheeling discharge of the same system with initial currents $100 \mathrm{~A}$ and $200 \mathrm{~A}$. Those

measurements, which are not included here, yielded the value of $L_{a}=.65 \mathrm{Henry}$, which deviates less then $10 \%$ from the effective inductance value measured under the real conditions.

\section{CONCLUSIONS}

The experiments yielded results which are significant for practical applications:

1. The resistance and inductance of the WAMUS magnet system for the purpose of calculating discharge time are $.058 \mathrm{ohms}$ and .78 henrys respectively.

2. The method of the freewheeling discharge of the analytical magnet with relatively low current (100A to $200 \mathrm{~A}$ ) provides an adequate effective inductance value to predict the discharge time of the magnet under actual working conditions.

3. To estimate the amount of energy stored in the magnetic field under the steady state working conditions, the best technique is to use the calculated steady state inductance of the magnet ( 1.72 Henry).

4. It is possible to reduce the current in the DO WAMUS magnet without harm to data taking and with substantial savings on electricity cost. However such a change would require recalibration of the system, which, has been determined to be unpractical during the current experiment run.

\section{ACKNOWLEDGMENT}

We would like to thank Stan Orr RD/EED for giving the initial impulse and influencing the direction of this work and also Edwin Arko RD/DZERO and Julius Lentz RD/EED for their help with the measurements. 


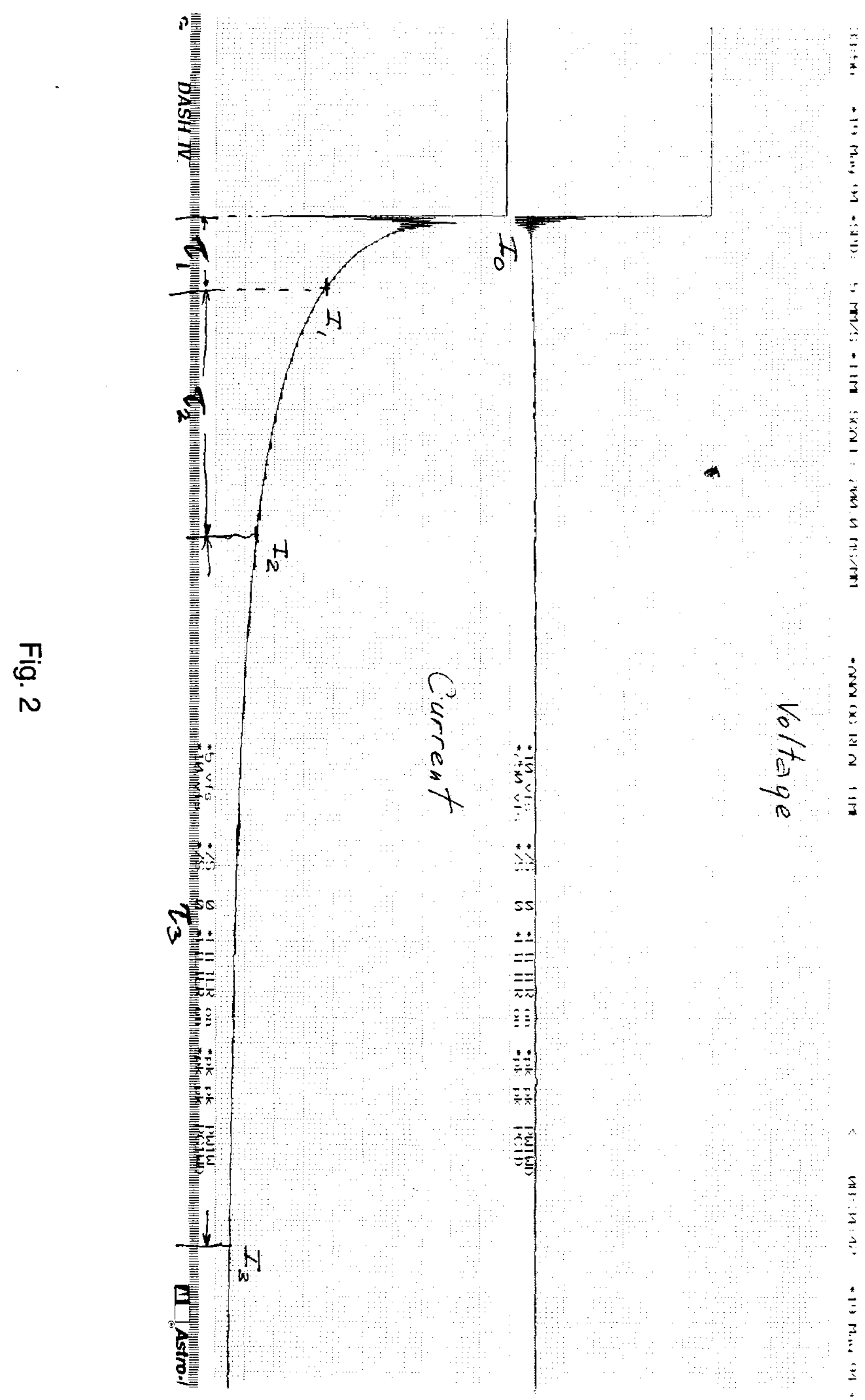

